\title{
THE NUMBER OF LOCALLY RESTRICTED DIRECTED GRAPHS ${ }^{1}$
}

\author{
LEO KATZ AND JAMES H. POWELL
}

1. Preliminaries. We shall be concerned with finite graphs of $t$ directed lines on $n$ points, or nodes. The lines are joins from one point to another. To each point, $P_{i}$, is associated a non-negative number pair $\left(r_{i}, s_{i}\right)$, where $r_{i}$ is the number of lines from $P_{i}$, and $s_{i}$ is the number of lines to $P_{i}$. These number pairs are considered to be fixed local restrictions. Obviously, $\sum_{i} r_{i}=\sum_{j} s_{j}=t$. The vectors $\boldsymbol{r}$ and $\mathrm{s}$, with elements $r_{i}$ and $s_{i}$, respectively, are two $n$-part non-negative integer partitions of $t$, with vanishing parts permitted.

In general, it is possible to construct many $n$-node graphs satisf ying a specified set of local restrictions. The main result of this paper gives the number of distinct graphs of this kind. The enumeration is helpful in studying communication networks in which the $i$ th element has $r_{i}$ receivers and $s_{i}$ senders; here the pertinent question is whether such graphs exist. In the isomorphic problem in group organization theory, where the $i$ th individual in the group gives information or orders or choices to $r_{i}$ others and receives from $s_{i}$ others, the exact number of graphs is needed in order to construct the probability distributions for random variables defined over the graphs. All of these activities, like the graphs, are irreflexive and take values on a twoelement Boolean algebra.

Previous work on numbers of graphs has been devoted exclusively to counts of graphs topologically distinct under permutations of the points which preserve joins. Thus, G. Pólya [9] gave explicitly the numbers of trees and rooted trees on $n$ points, R. Otter [8] gave simpler purely combinatorial methods for counting trees and rooted trees, and F. Harary and G. E. Uhlenbeck [6] gave the numbers of free "pure" and "mixed" Husimi trees. Also, F. Harary indicates in an abstract [5] that Polya's method of generating functions may be extended to give the numbers of ordinary and of directed graphs on $n$ points and $t$ lines. R. L. Davis [3] defines and gives a method for counting the numbers of various subclasses of directed graphs on $n$ points. The problem we face is essentially different from all of these and requires quite different methods of enumeration.

We shall make use of certain bipartitional functions due to Cayley

Presented to the Society, September 1, 1953; received by the editors July 27, 1953 and, in revised form, January 27, 1954.

1 This work was supported by the Office of Naval Research. 
[1]. We shall proceed by defining an operation on such partitions. Then, a first lemma gives Cayley's function in terms of repeated applications of this operation on numbers of the kind we require and a second provides an inverse for the operation; together, they supply the desired expression for the number of distinct directed graphs in terms of the known bipartitional functions of Cayley.

2. Representation of directed graph as matrix. König [7, pp. 141$143]$ gives two matric representations of directed graphs. One expresses incidence relations between points and lines, the other, incidence between lines and cycles; both are inappropriate for present purposes. We shall use instead a representation discussed for group organization theory by Forsyth and Katz [4].

In this representation, the graph of $t$ lines on $n$ points corresponds uniquely to a square matrix $C=\left(c_{i j}: n \times n\right)$, where $c_{i j}=1$ if, in the graph, a directed line connects the node $P_{i}$ to the node $P_{j}$; otherwise, $c_{i j}=0$. Obviously, every $c_{i i}=0, r_{i}=\sum_{k} c_{i k}$ is the number of joins starting from $P_{i}$, and $s_{i}=\sum_{k} c_{k i}$ is the number of joins ending on $P_{i}$. Also, $\sum_{i} r_{i}=\sum_{j} s_{j}=t$, the total number of lines. Then, the number of distinct graphs satisfying the specified local restrictions, $\eta(s, r)$, is identical with the number of distinct matrices, $C$, of zeros and ones, satisfying the three conditions:

(i) the row sums of $C$ are the vector $r$,

(ii) the column sums of $C$ are the vector $s$, and

(iii) the principal diagonal elements of $C$ are identically zero.

3. A relevant bipartitional function. P. V. Sukhatme [10] considered, among other problems, that of finding the number, $A(s, r)$, of possible ways in which the cells of a $\rho \times \sigma$ lattice can be filled with zeros and ones so that

(i) the column totals from left to right form the parts of the partition $s$ in some fixed order, and

(ii) the row totals from top to bottom form the parts of the partition $\boldsymbol{r}$ in some fixed order.

He pointed out that $A(s, r)=A(r, s)$. It is also evident that this number is not altered by addition of a sufficient number of rows or columns of zeros to make the lattice square. We may, therefore, take $n=\max (\rho, \sigma)$; then Sukhatme's matrices include those corresponding to our directed graphs as well as others which violate condition (iii) above. Our main theorem expresses $\eta(s, r)$ as a linear combination of the known $A\left(s_{\alpha}, r_{\alpha}\right)$. The fundamental idea of the theorem is to delete from $A(s, r)$ the number of matrices having some 1's on the principal diagonal. This was first accomplished by a process of alternate inclu- 
sion and exclusion. The theorem as given here represents a very considerable simplification of the original argument.

We complete the definitions of $\eta$ and $A$ with the conventions that both vanish identically if any index is negative and that both are linearly additive over the partitions.

Sukhatme $[10$, p. 389] gave tables of $A(P, Q)$ for $P, Q$ partitions of $t$ for $t=1,2, \cdots, 8$. These are identical with tables given by Cayley [1] in 1857 for certain symmetric functions for weights $t$ $=1,2, \cdots, 10$. Recently, David and Kendall [2] recomputed and extended these tables through $t=12$ and gave references to unpublished tables of weight 14 and 15 .

Sukhatme's paper $[10$, p. 387] gave, also, an algorithm for computation of a single $A(P, Q)$ of any weight. Thus implicitly, if not practically, our problem is completely solved when we express $\eta(s, r)$ in terms of $A\left(s_{\alpha}, r_{\alpha}\right)$.

4. The number of directed graphs. We shall require operators $\delta_{i}(i=1,2, \cdots, n)$ defined by

$$
\begin{aligned}
\delta_{i}\left(s_{1}, \cdots, s_{i}, \cdots, s_{n} ; r_{1}, \cdots, r_{i}, \cdots, r_{n}\right) & \\
& =\left(s_{1}, \cdots, s_{i}-1, \cdots, s_{n} ; r_{1}, \cdots, r_{i}-1, \cdots, r_{n}\right) .
\end{aligned}
$$

The effect of the operation $\delta_{i}$ is to replace the double partition $(s, r)$ of $t$ by a double partition of $(t-1)$ with the $i$ th part of each reduced by unity. If we take $\left(\delta_{i}+\delta_{j}\right)(s, r) \equiv \delta_{i}(s, r)+\delta_{j}(s, r)$, the obvious commutativity of the $\delta$ 's serves to define every sum of monomials of the form $\sum_{(\alpha)}\left(\prod_{1}^{n} \delta_{t}^{\alpha_{i}}\right)$ as an operator on $(s, r)$. We note that the number of nontrivial terms in the application of such an operator to $(s, r)$ is necessarily finite for finite $t$ since both $A\left[\delta_{i}^{m+k}(s, r)\right] \equiv 0$ and $\eta\left[\delta_{i}^{m+k}(s, r)\right] \equiv 0$ for $k>0$ and $m=\min \left(s_{i}, r_{i}\right)$. Finally, we observe that any identity in the $A\left(s_{\alpha}, r_{\alpha}\right)$ and $\eta\left(s_{\beta}, r_{\beta}\right)$ is unaffected by application of these operators to the partitions involved.

LEMMA I. If the vectors $s$ and $\boldsymbol{r}$ are any two $n$-part ordered partitions of $t$, then

$$
A(s, r)=\eta\left\{\prod_{1}^{n}\left(1+\delta_{i}\right)(s, r)\right\}
$$

Proof. Every matrix belonging to the set enumerated by $A(s, r)$ has either no principal diagonal elements different from zero, or one specified p.d. element different from zero, or two specified such elements, etc. This exhaustive disjunction of the set gives subsets each isomorphic with a set enumerated by an $\eta\left(s_{\alpha}, r_{\alpha}\right)$ where the vectors 
$\boldsymbol{s}_{\boldsymbol{\alpha}}$ and $\boldsymbol{r}_{\alpha}$ are formed from $\boldsymbol{s}$ and $\boldsymbol{r}$ by reduction by unity of each component corresponding to a specified p.d. element different from zero. Then,

$$
\begin{aligned}
A(s, r)= & \eta(s, r)+\sum_{i} \eta\left[\delta_{i}(s, r)\right]+\sum_{i<i} \eta\left[\delta_{i} \delta_{j}(s, r)\right] \\
& +\cdots+\eta\left[\delta_{1} \delta_{2} \cdots \delta_{n}(s, r)\right] \\
= & \eta\left\{\prod_{1}^{n}\left(1+\delta_{i}\right)(s, r)\right\} .
\end{aligned}
$$

Lemma II. For $s$ and $\boldsymbol{r}$ as in the preceding lemma, the operator $\left(1+\delta_{i}\right)$ has an inverse (right and left) given by

$$
\left(1+\delta_{i}\right)^{-1} \equiv 1-\delta_{i}+\delta_{i}^{2}-\delta_{i}^{3}+\cdots .
$$

Proof. $\left(1+\delta_{i}\right)^{-1}\left(1+\delta_{i}\right)(s, r)=\left(1+\delta_{i}\right)^{-1}\left[(s, r)+\left(s-u_{i}, r-u_{i}\right)\right]$, where $\boldsymbol{u}_{i}$ is a vector with $i$ th component unity and all others zero. Next,

$$
\begin{aligned}
\left(1+\delta_{i}\right)^{-1}[(s, r) & \left.+\left(s-u_{i}, r-u_{i}\right)\right] \\
= & (s, r)-\left(s-u_{i}, r-u_{i}\right)+\left(s-2 u_{i}, r-2 u_{i}\right)-\cdots \\
& +\left(s-u_{i}, r-u_{i}\right)-\left(s-2 u_{i}, r-2 u_{i}\right)+\cdots,
\end{aligned}
$$

and $\left(1+\delta_{i}\right)^{-1}$ is a left inverse. It is equally easily shown that $\left(1+\delta_{i}\right)^{-1}$ is a right inverse.

Repeated application of (3) to both members of (2) gives immediately the

Theorem. $\eta(s, r)=A\left\{\prod_{1}^{n}\left(1+\delta_{i}\right)^{-1}(s, r)\right\}$.

In application of the theorem, and to a lesser extent in application of Lemma I, we note that the series in (3) may be terminated at the $m_{i}$ th power, where $m_{i}=\min \left(r_{i}, s_{i}\right)$, since the functions $A\left(s_{\alpha}, r_{\alpha}\right)$ evaluated beyond this point all vanish. Thus, in every case, $\eta(s, r)$ is evaluated by additions and subtractions of finitely many $A\left(s_{\alpha}, r_{\alpha}\right)$.

5. Examples. We wish to compute the number of directed graphs on four points where the numbers of lines in and out of the points are $(3,2),(2,1),(1,2)$, and $(1,2)$, respectively; i.e., we wish to compute $\eta(3,2,1,1 ; 2,1,2,2)$. The remark following the theorem gives us $\eta(3,2,1,1 ; 2,1,2,2)=\left(1-\delta_{1}+\delta_{1}^{2}\right)\left(1-\delta_{2}\right)\left(1-\delta_{3}\right)\left(1-\delta_{4}\right) A(3,2,1,1$; $2,1,2,2)$. Sukhatme's tables for $t=7,6, \cdots, 2$ give the required numbers.

Operating as indicated on the right-hand member of the equation above, we have 


$$
\begin{aligned}
\eta(3,2,11 ; 2,1,2,2)= & A\left(3,2,1^{2} ; 2^{3}, 1\right)-A\left(2^{2}, 1^{2} ; 2^{2}, 1^{2}\right) \\
& -A\left(3,1^{3} ; 2^{3}\right)-2 A\left(3,2,1 ; 2^{2}, 1^{2}\right) \\
& +2 A\left(2,1^{3} ; 2^{2}, 1\right)+2 A\left(2^{2}, 1 ; 2,1^{3}\right) \\
& +2 A\left(3,1^{2} ; 2^{2}, 1\right)+A\left(3,2 ; 2,1^{3}\right) \\
& -A\left(3,1 ; 2,1^{2}\right)-A\left(2^{2} ; 1^{4}\right)-2 A\left(2,1^{2} ; 2,1^{2}\right) \\
& -A\left(1^{4} ; 2^{2}\right)-2 A\left(2,1^{2} ; 2,1^{2}\right)+A\left(2,1 ; 1^{3}\right) \\
& +A\left(2,1 ; 1^{3}\right)+2 A\left(1^{3} ; 2,1\right)-A\left(1^{2} ; 1^{2}\right),
\end{aligned}
$$

where the terms on the right are, by groups, of weight $7,6, \cdots, 2$, respectively. The sum of the 12 positive terms is 94 , the 12 negative terms total 91 , and $\eta(3,2,1,1 ; 2,1,2,2)=3$. The three graphs, in matrix representation and graph form, are:

$$
\left[\begin{array}{llll}
0 & 0 & 1 & 1 \\
1 & 0 & 0 & 0 \\
1 & 1 & 0 & 0 \\
1 & 1 & 0 & 0
\end{array}\right], \quad\left[\begin{array}{llll}
0 & 1 & 1 & 0 \\
1 & 0 & 0 & 0 \\
1 & 0 & 0 & 1 \\
1 & 1 & 0 & 0
\end{array}\right], \quad\left[\begin{array}{llll}
0 & 1 & 0 & 1 \\
1 & 0 & 0 & 0 \\
1 & 1 & 0 & 0 \\
1 & 0 & 1 & 0
\end{array}\right]
$$

and
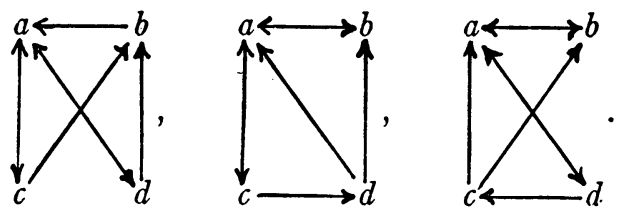

Obviously, in this case, the three graphs could have been exhibited directly and the method seems cumbersome. By way of contrast, 16 positive and 16 negative terms give $\eta(3,2,1,1,1,0,0,0 ; 1,1,1,1$, $1,1,1,1)=4846-3703=1143$; it is no longer feasible to exhibit the distinct directed graphs on these eight points.

6. Remark. It would be of interest to compute the number of ordinary graphs (not directed) subject to pointwise local restrictions on the joins. So far, we have been unable to do so.

\section{REFERENCES}

1. A. Cayley, A memoir on the symmetric functions of the roots of an equation, Philos. Trans. Roy. Soc. London. Ser. A vol. 147 (1857) pp. 489-499.

2. F. N. David and M. G. Kendall, Tables of symmetric functions. Parts II and III, Biometrika vol. 38 (1951) pp. 435-462.

3. R. L. Davis, The number of structures of finite relations, Proc. Amer. Math. Soc. vol. 4 (1953) pp. 486-495. 
4. E. Forsyth and L. Katz, A matrix approach to the analysis of sociometric data, Sociometry vol. 9 (1946) pp. 340-347.

5. F. Harary, The number of graphs and directed graphs, Bull. Amer. Math. Soc. Abstract 58-6-717.

6. F. Harary and G. E. Uhlenbeck, On the number of Husimi trees, I, Proc. Nat. Acad. Sci. U.S.A. vol. 39 (1953) pp. 315-322.

7. D. König, Theorie der endlichen und unendlichen Graphen, Leipzig, 1936.

8. R. Otter, The number of trees, Ann. of Math. vol. 49 (1948) pp. 583-599.

9. G. Pólya, Kombinatorische Anzahlbestimmungen für Gruppen, Graphen und chemische Verbindungen, Acta Math. vol. 68 (1937) pp. 145-254.

10. P. V. Sukhatme, On bipartitional functions, Philos. Trans. Roy. Soc. London. Ser. A. vol. 237 (1938) pp. 375-409.

University of California, Berkeley, and

Michigan State College 